\title{
A importância dos jogos didáticos no processo de ensino aprendizagem: Revisão
}

\section{integrativa}

\author{
The importance of didactic games in the teaching-learning process: An integrative review \\ La importancia de los juegos didácticos en el processo de enseñanza-aprendizaje: una revisión
}

integradora

Recebido: 26/03/2021 | Revisado: 31/03/2021 | Aceito: 06/04/2021 | Publicado: 16/04/2021

Franciane Silva Almeida

ORCID: https://orcid.org/0000-0002-7894-5712 Instituto Federal de Educação, Ciência e Tecnologia de Minas Gerais, Brasil E-mail: francy.sa.meida@gmail.com

Patrícia Batista de Oliveira

ORCID: https://orcid.org/0000-0001-6982-1761 Instituto Federal de Educação, Ciência e Tecnologia de Minas Gerais, Brasil E-mail: patricia.bio77@gmail.com

Deyse Almeida dos Reis

ORCID: https://orcid.org/0000-0002-6627-1247 Instituto Nacional da Mata Atlântica, Brasil E-mail: deysereis.reis@gmail.com

\begin{abstract}
Resumo
Jogos lúdicos são metodologias úteis para os processos de ensino-aprendizagem, pois se caracterizam como alternativas que auxiliam a construção de conhecimento pelos alunos. Os jogos eas brincadeiras vêm ganhando espaço e importância em todas as abordagens referentes à infância. A atividade lúdica, que pode se expressar no jogo, no brinquedo ou brincadeira, tem importância fundamental na educação escolar e na formação do homem. Ela permite ao educador perceber traços da personalidade e do comportamento do educando, o que facilita o planejamento de estratégias pedagógicas no ambiente lúdico, promovendo a motivação para uma melhor aprendizagem. O objetivo desta pesquisa foi realizar uma revisão integrativa em plataformas bibliográficas para verificar a eficácia do uso de jogos educacionais no ambiente escolar, buscar também a importância do uso de jogos educativos para o auxílio do profissional docente e identificar o aprendizado dos discentes através de jogos didáticos. Em resultados da revisão foram encontradas 09 publicações no período de 10 anos nos anos de 2010 a 2020. As publicações abordam como a atividade lúdica é essencial no âmbito escolar e como os docentes necessitam de mais recursos e motivação pela escola para melhor aplicação do mesmo.
\end{abstract}

Palavras-chave: Brincadeira; Conhecimento; Escola; Aluno; Ensino.

\begin{abstract}
Playful games are useful methodologies for teaching-learning processes, as they are characterized as alternatives that help students build knowledge. Games and games are gaining space and importance in all approaches related to childhood. The playful activity, which can be expressed in the game, in the toy or play, is of fundamental importance in school education and in the training of man. It allows the educator to perceive traits of the student's personality and behavior, which facilitates the planning of pedagogical strategies in the ludic environment, promoting motivation for better learning. The objective of the work was to carry out an integrative review in bibliographic platforms to obtain results of the effectiveness of the use of educational games in the school environment, also to seek the importance of the use of educational games to help the teaching professional and to identify the students' learning through educational games. In the results of the review, 9 publications were found over a 10-year period from 2010 to 2020. The publications address how playful activity is essential in the school environment and how teachers need more resources andmotivation by the school to better apply it.
\end{abstract}

Keywords: Play; Knowledge; School; Student; Teaching.

\section{Resumen}

Los juegos lúdicos son metodologías útiles para los procesos de enseñanza-aprendizaje, ya que se caracterizan como alternativas que ayudan a los estudiantes a construir conocimientos. Losjuegos y los juegos están ganando espacio e importancia en todos los enfoques relacionados con la infancia. La actividad lúdica, que puede expresarse en el juego, en el juguete o el juego, es de fundamental importancia en la educación escolar y en la formación del hombre. Permite al educadorpercibir rasgos de la personalidad y comportamiento del alumno, lo 
que facilita la planificación de estrategias pedagógicas en el entorno lúdico, promoviendo la motivación para un mejor aprendizaje.El objetivo del trabajo fue realizar una revisión integradora en plataformas bibliográficas para obtener resultados de la efectividad del uso de juegos educativos en el ámbito escolar, así como buscar la importancia del uso de juegos educativos para ayudar al profesional docente y a identificar el aprendizaje de los estudiantes a través de juegos educativos. En los resultados de la revisión, se encontraron 9 publicaciones durante un período de 10 años de 2010 a 2020. Las publicaciones abordan cómo la actividad lúdica es esencial en el entorno escolar y cómo los maestros necesitan mas recursos y motivación por parte de la escuela para aplicarla mejor.

Palabras clave: Juego; Conocimiento, Escuela, Estudiante; Enseñanza.

\section{Introdução}

Historicamente o modelo utilizado na formação dos profissionais de educação tem sido traçado de acordo com as metodologias de ensino-aprendizagem tradicionais, denominado por PauloFreire como bancária, pelo fato de apenas ocorrer a transferência de conhecimento pelo docente ao aluno, não se mostra suficiente para atender às múltiplas necessidades dos indivíduos e da formação dos profissionais (Freire, 2011).

No recente contexto social, observa-se que a percepção do mundo se modifica a cada momento, sendo uma rede dinâmica que sofre inúmeras transformações, desta forma as instituições de ensino têm discutido a urgente necessidade de reeditar seu papel social e o processo de ensino- aprendizado ainda vigente, devendo estimular cada vez mais o pensamento crítico e a autonomia do estudante. (Marin, Güllich, 2015; Miranda et al., 2016).

A lei de Diretrizes e Bases da Educação Nacional (LDB) e as Diretrizes Curriculares Nacionais (DCN) incentivam nas instituições formadoras buscarem por um modelo que possibilite a aproximação da realidade social, incitando o aluno a refletir sobre o meio que vive e possuir um raciocínio crítico a fim de buscar meios que possam transformar seu contexto. Nessa perspectiva de mudanças no processo de formação dos estudantes há destaque para as Metodologias Ativas de Aprendizagem, tendo como modo de operacionalização a "Problematização" e "Aprendizagem Baseada em Problemas” (ABP) (Cyrino, Toralles-Pereira, 2004; Marin, Güllich, 2015).

Na Aprendizagem Baseada em Problemas (APB) há formação de grupos de alunos, onde o docente apresenta problemas pré-elaborados, a base para escolha dos temas está descrita na grade curricular do curso, o aluno deverá dominar e conhecer previamente o tema abordado na situação para que ocorra a devido discussão em grupo. Essa metodologia proporciona a integração das disciplinas (Cyrino \& Toralles-Pereira, 2004).

A Aprendizagem Baseada em Problemas (ABP) possui marcas de sua origem na Teoria da Indagação de John Dewey, filósofo, psicólogo e educador, para ele a forma de aprender deve partir de desafios, problemas ou situações intencionais que provoquem dúvidas (Salido López, 2020). Partindo deste ponto esse método de ensino realçaria a descoberta, a vivência e a reflexão. Os docentes lançariam determinado problema ou questionamento de acordo com os conteúdos programáticos. (Marin, Güllich, 2015). No mesmo patamar encontra-se a aprendizagem baseada em jogos e brincadeiras que contribuem para o desenvolvimento de habilidades sociais, emocionais e cognitivas do aluno (Pinto et al., 2021). O jogo didático deve ser utilizado como forma de simplificar ou até mesmo como um meio de associar o conteúdo trabalhado em sala de aula com algo mais "palpável" e atrativo aos discentes. Pode ser utilizado para atingir determinados objetivos pedagógicos (Do canto et al., 2021). Outro aspecto não menos relevante, um dos maiores desafios do dia a dia do professor é transformar o aprendizado em uma tarefa lúdica. Para isso, não é preciso apenas muita criatividade e jogo de cintura, mas também instrumentos que atendam as necessidades pedagógicas dos alunos e atraiam o interesse deles. Jogos e brincadeiras sãoperfeitos para isso. Os jogos ajudam no desenvolvimento do aluno sob as perspectivas criativa, afetiva, histórica, social e cultural (Dos santos et al., 2020).

Nesse diapasão, o uso de ferramentas, como os jogos, tem como função preencher aquelas lacunas deixadas pelos professores como resultado de uma educação engessada que presenciamos hoje em dia. Isto é, a aplicação de um 
jogo didático é extremamente positiva na medida em que favorece a socialização com os colegas, além de contribuir para a construção de conhecimentos novos e mais elaborados (Ferreira et al, 2020).

Não obstante, os jogos didáticos podem ser planejados e organizados de inúmeras formas, porém algumas ações são fundamentais. Exemplificando, para aplicação do recurso metodológico é necessária delimitação de um tema como biologia celular, botânica e outros. Em seguida, ter clareza de que as crianças e/ou adolescentes possuem conhecimento prévio(subsunçores) sobre o conteúdo trabalhado. Por conseguinte, selecionar previamente os recursos e materiais que serão usados.

Voltando às concepções iniciais, o professor tem o papel de deixar claro para a sala de aula os objetivos educacionais do jogo didático, ou seja, com o planejamento das etapas, o docente irá abordar uma complexidade crescente antecipando quais serão as perguntas que fará para encaminhar a atividade. Ademais, prever quais momentos será em grupo, em duplas e individuais, como também estabelecer os critérios de avaliação e registrar a participação de cada um ao longo do jogo didático.

Em suma, este estudo tem como objetivo realizar revisão integrativa em plataformas bibliográficas para termos resultados da eficácia do uso de jogos educacionais no ambiente escolar, como também, buscar a importância do uso de jogos educativos para o auxílio do profissional docentee identificar o aprendizado dos discentes através de jogos didáticos.

\section{Metodologia}

Trata-se de uma revisão integrativa da literatura, definida como a mais ampla abordagem metodológica referente às revisões para uma compreensão completa do fenômeno analisado. Este método de pesquisa tem por finalidade reunir e sintetizar resultados de pesquisas sobre um delimitado tema, de maneira sistemática e ordenada, contribuindo para o aprofundamento do conhecimento do tema investigado (Mendes, 2008).

Para elaboração desta revisão foram percorridas as seguintes etapas: definição da questão de pesquisa e objetivos da revisão; estabelecimento de critérios de inclusão e exclusão dos artigos, leitura dos títulos; leitura dos resumos, seleção das informações a serem extraídas dos estudos selecionados; análise dos resultados; interpretação e discussão dos resultados. (Mendes, 2008). A última etapa foi constituída pela apresentação da revisão no formato de artigo científico.

A pesquisa foi realizada nos períodos de 01 de julho a 30 de julho de 2020 nas seguintes bases de dados: SciELO (Scientific Electronic Library Online) e Google acadêmico. Mediante o cruzamento dos seguintes descritores: "Ludicidade", "Jogo didático", "Jogos educativos", "Jogos no ensino de biologia", "Jogos no ensino de ciências", "Projetos baseados em problemas","Ensino-Aprendizagem".

O recorte temporal adotado foram artigos publicados nos últimos 10 anos, os quais correspondem aos anos de 2010 a 2020. Para a análise realizou-se uma leitura crítica dos estudos,procedida da extração dos dados de interesse para a revisão e do preenchimento do quadro sinóptico, conforme a Tabela 2. Este quadro foi organizado em número do artigo, nome dos autores/ano de publicação, título, base de dados, tipos de pesquisa e periódico.

O profissional docente requer cuidados necessários com a elaboração e execução de jogos didáticos no ambiente escolar, com também, as consequências na vida do educando. Portanto, como embasamento teórico elaboramos a seguinte pergunta norteadora para guiar a revisão integrativa:“O jogo didático é um instrumento facilitador para prática docente?”. Sendo assim, o presente estudo fará uma revisão na produção científica para fornecer uma compreensão mais abrangente do fenômeno de usos de jogos educacionais.

Os critérios de inclusão foram: artigos e monografias originais e confiáveis disponibilizados na íntegra eletronicamente e de forma gratuita, artigos e revistas nos idiomas em português, pesquisas relacionadas com jogos 
educacionais e artigos que respondessem à pergunta norteadora. Foram excluídos: artigos incompletos, artigos em outros idiomas e repetidos, pesquisas que não incluem o uso de materiais alternativos como recurso didático.

\section{Resultados e Discussão}

$\mathrm{Na}$ análise inicial foram identificados 50 artigos. Em seguida, os títulos e resumos foram lidos restando 25 artigos para leitura na íntegra. Seguindo os critérios de inclusão e exclusão 09 artigos compuseram o número amostral. Para maior compreensão, na Figura 1, é ilustrado o procedimento de seleção dos artigos que formaram a amostra da revisão integrativa.

Figura 1. Fluxograma dos trabalhos científicos encontrados nas bases de dados on-line.

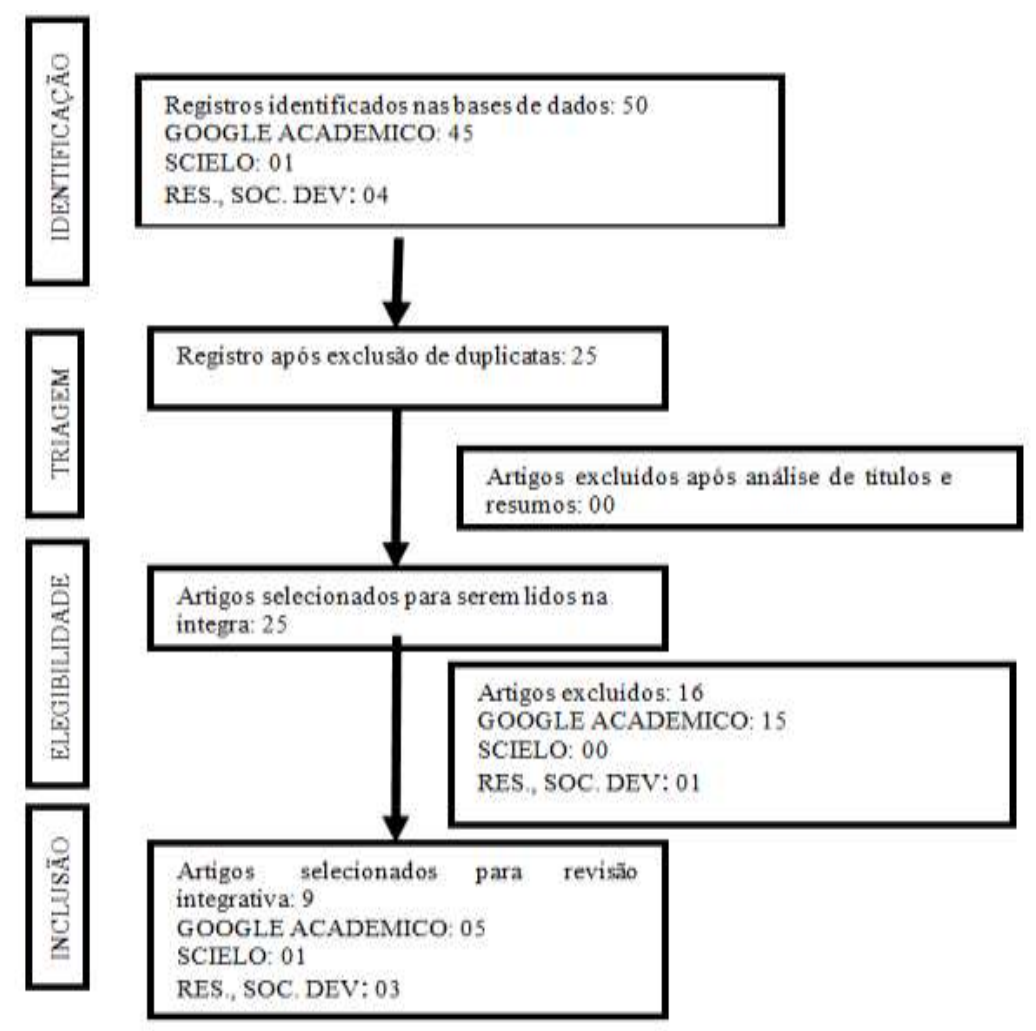

Fonte: Autoras.

Dentro do período analisado percebeu-se uma quantidade maior de estudos para o ano de 2020 e 2013. Nesta busca não foram encontrados artigos publicados nos anos de 2011, 2012, 2015, 2016 e 2017 (Tabela 1). 
Tabela 1. Distribuição dos estudos por ano de publicação.

\begin{tabular}{c|c}
\hline $\begin{array}{c}\text { ANO DE } \\
\text { PUBLICAÇÃO }\end{array}$ & NÚMERO DE ESTUDOS \\
\hline 2010 & 01 \\
\hline 2011 & 00 \\
\hline 2012 & 00 \\
\hline 2013 & 03 \\
\hline 2014 & 01 \\
\hline 2015 & 00 \\
\hline 2016 & 00 \\
\hline 2017 & 00 \\
\hline 2018 & 01 \\
\hline 2019 & 01 \\
\hline 2020 & 02 \\
\hline TOTAL & $\mathbf{0 9}$ \\
\hline
\end{tabular}

Fonte: Autoras.

Tabela 2. Resumo dos trabalhos científicos encontrados nas bases de dados on-line.

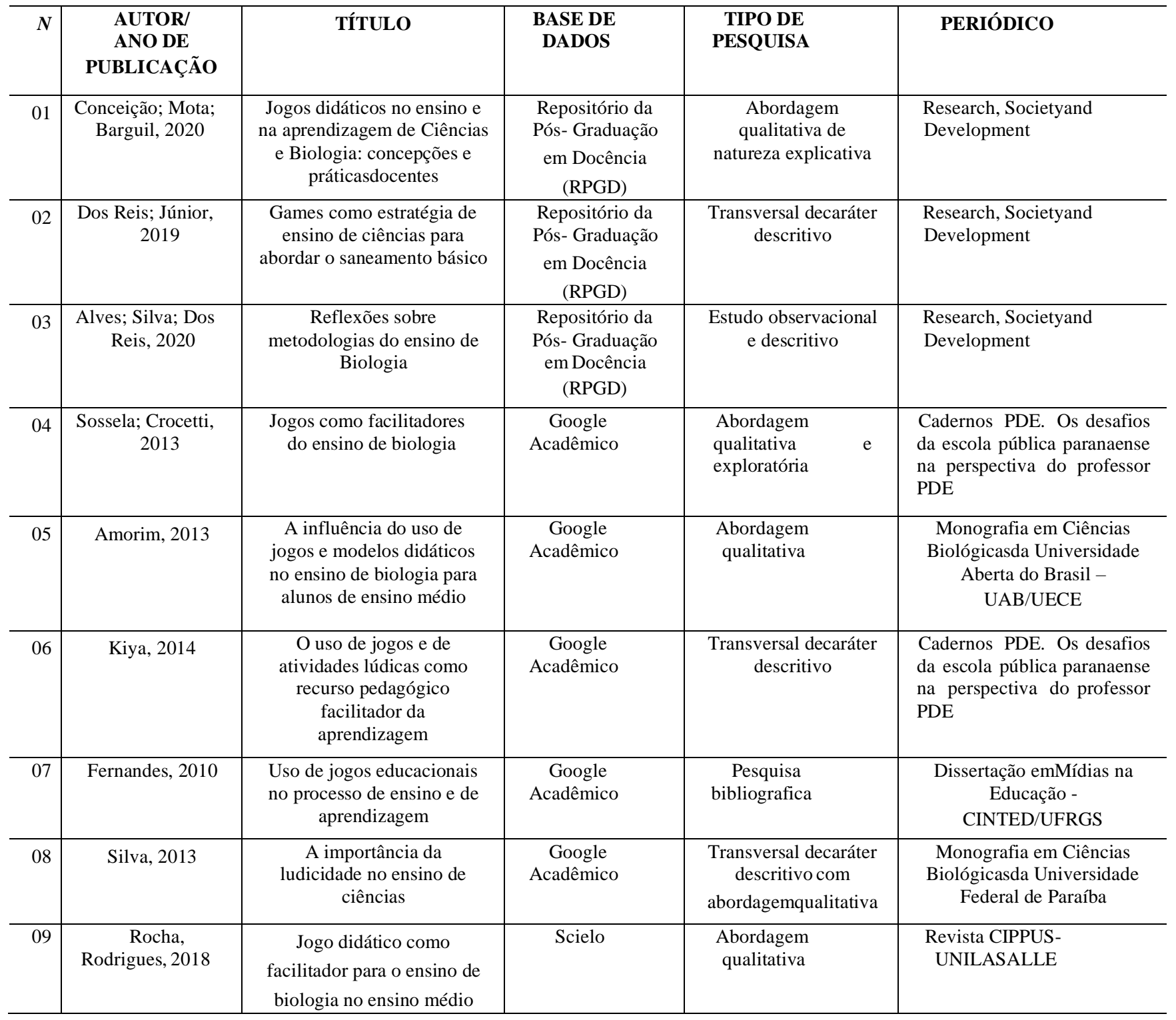


Nossos resultados (Tabela 2) demonstram que entre um período de 10 anos, 09 publicações foram encontradas. Considerando a presença do lúdico em sala de aula podemos afirmar que essa ferramenta pedagógica é de extrema importância no processo de ensino-aprendizagem dos alunos, pois funciona como exercícios necessários e úteis, sendo as brincadeiras e jogos elementos indispensáveis para que haja uma aprendizagem com divertimento, que proporcione o prazer no ato de aprender, e que facilite as práticas pedagógicas em sala de aula (Salomão \& Martini, 2007).Com todos esses fatores positivos que podem ser adquiridos através do lúdico ainda é preciso inserir essa metodologia de forma mais presente nas escolas, todavia, a quantidade de publicações realizadas durante esses 10 anos nos mostra um número relativamente baixo que consequentemente remete que o uso dessas práticas no ambiente escolar não ocorre com frequência. De acordo com essas publicações realizadas durante esses 10 anos, vários autores demonstraram em diferentes visões a importância do jogo lúdico.

Fernandes (2010) em seu trabalho com jogos digitais procurou buscar como esses mecanismos estão inseridos no trabalho pedagógico. O autor acredita que os jogos, sob a visão da criança, constituem a maneira mais divertida e interessante de aprender e oferecem ao professor a oportunidade de observar e analisar a forma como a criança aprende. Afirma também que as inovações tecnológicas não podem ser ignoradas pelas escolas e professores em que a grandeza lúdicado jogo proporciona um instrumento amplo no processo de ensino e aprendizagem, pois permite de forma dinâmica o desenvolvimento de aspectos relacionados a áreas cognitivas, afetiva, social, linguística e motora, entre outras. Já Silva (2013) partiu do objetivo de analisar os resultados de uma intervenção pedagógica utilizando jogos lúdicos como forma de auxiliar no processo de ensino- aprendizagem de Ciências em turmas de $6^{\circ}$ ano do Ensino Fundamental. E por aliar os aspectos lúdicos aos cognitivos, o autor questiona que o jogo é uma importante estratégia para o ensino e a aprendizagem de conceitos abstratos e complexos, favorecendo a motivação interna, o raciocínio, a argumentação, a interação entre alunos e entre professores e alunos (Silva, 2013). Deste modo, ele pode observar que de acordo com seus resultados analisados nesta pesquisa as turmas onde os alunos tiveram a oportunidade de estudar os conteúdos de Ciências com o auxílio dos jogos lúdicos foram registrados maiores índices de aprendizado em torno de $80 \%$, enquanto que as turmas onde não ocorreram as atividades lúdicas obteve um índice bastante inferior em torno de 30\%. Demonstrando então que o lúdico apresenta influência no momento de aprendizado.

Tratando de influência, Amorim (2013) levanta um questionamento no decorrer do seu trabalho sobre o lúdico, com: “Os jogos e modelos didáticos influenciam a prática pedagógica doprofessor de Biologia no Ensino Médio?”. Após buscas através de pesquisas direto com os professores, em seu trabalho Amorim pode concluir em seu questionamento que existe sim influências significativas na utilização dos jogos e modelos didáticos na transmissão de conteúdos ligados a Biologia, estes podem completar as lacunas existentes no aprendizado dos estudantes que apresentam dificuldades em relacionar os conceitos estruturais e funcionais biológicos com as imagens ilustrativas do livro. São instrumentos, segundo os professores entrevistados, que deixam a aula mais atraente e participativa, os alunos sentem-se instigados e se mostram questionar mais, favorece o contato com algo mais concreto, além de estimular a observação e socialização do que foi aprendido. Em contrapartida, Sossela e Crocetti (2013) abordaram o lúdico em sua pesquisa com o tema: "Jogos como facilitadores do ensino de biologia". Com base nas atividades ministradas nesta pesquisa os autores puderam observar que a maioria dos professores responsável pela disciplina de biologia sabe da ação dessa ferramenta metodológica, porém um dos motivos de não ter hábito de utilização desse método contínuo é pelo fato da carga horária que a disciplina de biologia exerce.

Relativamente uma carga horária baixa que nem sempre seria possível o uso do lúdico devido ao tempo. A metodologia do jogo, portanto, é uma forma interessante e desafiadora, pois visa a socialização entre os alunos, o desenvolvimento de atividades em grupos, a cooperação, a investigação, a busca pela resolução de problemas, o 
desenvolvimento do senso crítico, o entendimento e a compreensão de diversos tópicos. Kiya (2014) também traz a atividade lúdica como recurso pedagógico facilitador da aprendizagem. O autor preparou um caderno/estudo teórico sobre o uso da ludicidade através de jogos como uma forma de tornar o processo ensino e a aprendizagem mais interessante e prazerosa. O material elaborado buscou através de pesquisas subsídios teóricos que reforcem a importância e as diferentes possibilidades do uso de jogos como recurso pedagógico, facilitador do processo de ensino e aprendizagem. Contribuindo para que haja uma melhoria na prática educativa realizada nas escolas, e que professores e alunos possam vivenciar o processo de ensino e aprendizagem de forma mais dinâmica e prazerosa.

Concomitante Rocha e Rodrigues (2018) definindo o lúdico como facilitador de ensino visam demonstrar as etapas para a elaboração de um jogo didático-pedagógico nos moldes do banco imobiliário. Fundamentado em seus resultados, o jogo demonstrou-se eficaz, devido ao conhecimento assimilado pelos alunos onde foi construído de uma forma conjunta, de forma coesa, agradável, descontraída e dialógica. Obteve competitividade que surgiu durante a dinâmica, porém a mesma se mostrou positiva, estimulando o envolvimento dos alunos com o jogo. O trabalho também aborda o custo benefício do jogo, justificando que o mesmo além de possuir um baixo custo e fácil aplicação, pode ser usado de maneira a reforçar e fixar os conteúdos abordados em sala de aula.

Dos Reis e Júnior (2019) obtiveram a exploração do lúdico através do mundo do game. Foi proposto em seus estudos o uso das Tecnologias de Informação e Comunicação como ferramentas de ensino. Portanto, com a utilização da tecnologia apostaram nos jogos educativos eletrônicos abordando o tema Saneamento básico. Os autores selecionaram três jogos educativos eletrônicos disponíveis gratuitamente na internet: Caça Palavras (J1), Show Chuá (J2) e Trata City (J3). Cada jogo obteve um resultado: J1 "bom”, J2 "ótimo" e J3 "ótimo". Conforme esses resultados obtidos e discutidos observou-se que os jogos educativos selecionados auxiliaram no aprendizado e no desenvolvimento de competências para melhorar o rendimento dos alunos nas atividades escolares.

Embora a literatura traga todos esses resultados positivos sobre o lúdico, o parecer dos docentes sobre essa metodologia é bastante variado. Conceição et al. (2020) buscaram compreender as concepções de professores de Ciências e Biologia sobre a utilização dos jogos didáticos no contexto escolar. Em sua pesquisa os autores questionam sobre a aula expositiva, dizendo que a mesma sendo amplamente utilizada nas salas de aula, sozinha já não consegue atender e favorecer as diferentes habilidades dos estudantes. Como já foi dito, o jogo didático auxilia em diferentes aspectos, maximizando o desenvolvimento dos indivíduos e a construção do conhecimento. Deste modo, é importante que o professor esteja disposto ao uso dessa ferramenta, todavia, durante a realização desta pesquisa, foi possível constatar que, infelizmente, os professores ainda se limitam a utilizar estratégias didáticas convencionais, mesmo reconhecendo a importância da diversificação metodológica. Alves et al. (2020) também analisando a concepção dos professores, percebeu-se que as condições das escolas é um dos fatores que contribui para o insucesso desses métodos. A escola não proporciona esses momentos de reflexão sobre a prática docente, embora, para se desfrutar de um ensino prazeroso, não dependa unicamente de estruturas. Assim, os docentes necessitam elaborar mecanismos práticos para contextualização desses métodos, viabilizando, assim, a facilidade da aprendizagem e otimizando a realização de aulas participativas para os alunos. Tendo em vista todas essas questões comprobatórias sobre o lúdico, entendemos que o jogo é uma importante estratégia para o ensino e a aprendizagem de conceitos abstratos e complexos, favorecendo a motivação de alunos e professores, contudo, o jogo deveria merecer um espaço e um tempo maior na prática pedagógica cotidiana dos docentes.

\section{Considerações Finais}

Pelo exposto na literatura, compreendemos que o jogo é uma atividade lúdica eficaz que surge primeiramente sob a forma de simples exercícios motores, dependendo da sua realização apenas da maturação do aparelho motor. Sua 
finalidade é somente o próprio prazer do funcionamento. A criança vive em um mundo imaginário e quando chega ao ambiente escolar ela se depara com o novo, onde tudo é diferente. Desta forma "cabe ao professor proporcionar um ambiente agradável que facilite a adaptação da criança, uma vez que a transição resulta em um impacto muito grande e, por isso mesmo, exigirá, tanto do professor como dos pais, grande compreensão e paciência". Que a revisão integrativa realizada nesta pesquisa, não apenas contribua para informação de conhecimentos, mas também para sensibilizar os professores para a importância desses materiais. Concomitante, espera-se que trabalhos futuros possam ser realizados através da conscientização de professores sobre a importância de jogos didáticos estimulando-se a prática dessa metodologia como um viés facilitador de ensino aprendizado.

\section{Referências}

Alves, J. F., da Silva, L. B., \& dos Reis, D. A. (2020). Reflexões sobre metodologias do ensino de Biologia. Research, Society and Development, 9(8), e850985951-e850985951.

Amorim, A. D. S. (2013). A influência do uso de jogos e modelos didáticos no ensino de biologia para alunos de ensino médio. Monografia. Universidade Estadual do Ceará-UECE, Universidade Aberta do Brasil-UAB. Centro De Ciências e Saúde-CCS, Curso de Licenciatura em Ciências Biológicas. BeberibeCeará.

Cyrino, E. G., \& Toralles-Pereira, M. L. (2004). Trabalhando com estratégias de ensino-aprendizado por descoberta na área da saúde: a problematização e a aprendizagem baseada em problemas. Cadernos de Saúde Pública, 20, 780-788.

Da Conceição, A. R., Mota, M. D. A., \& Barguil, P. M. (2020). Jogos didáticos no ensino e na aprendizagem de Ciências e Biologia: concepções e práticas docentes. Research, Society and Development, 9(5), e165953290-e165953290.

Da rocha, D. F., \& Rodrigues, M. Da S. Jogo didático como facilitador para o ensino de biologia no ensino médio. Cippus, 6(2), 01-08, 2018.

Do Canto, C. G. D. S., Nunes, P. O. C., \& da Silva Rodrigues, A. C. (2021). O lúdico como ferramenta de aprendizagem de leitu ra e escrita. Revista eletrônica pesquiseduca, 13(29), 284-299.

Dos Reis, D. A., \& Júnior, N. V. (2019). Games como estratégia de ensino de ciências para abordar o saneamento básico. Research, Society and Development, $8(12)$, e428121846-e428121846.

Dos Santos, A. C., de Oliveira Santos, J., \& de Brito Araujo, M. J. (2020). Lúdico como ferramenta da psicopedagogia no desenvolvimento integral das crianças. Educte: Revista Científica do Instituto Federal de Alagoas, 10(1), 1175-1183.

Fernandes, N. A. (2010). Uso de jogos educacionais no processo de ensino e de aprendizagem. Monografia. Universidade Federal do Rio Grande do Sul CINTED/UFRGS. Centro Interdisciplinar de Novas Tecnologias na Educação.

Ferreira, S. M., Nascimento, C., \& Pitta, A. P. (2020). Jogos didáticos como estratégia para construção do conhecimento: uma experiência com o $6^{\circ}$ ano do Ensino Fundamental. Giramundo: Revista de Geografia do Colégio Pedro II, 5(9), 87-94.

Freire, P. (2011). Pedagogia da autonomia. São Paulo: Paz e Terra; 2001. Participação dos autores Rodrigo Caetano participou da concepção, organização e execução da pesquisa, além da redação final do texto. Volnei Garrafa, como orientador, participou de todas as etapas da pesquisa. Recebido, 16.

Freire, P. (2014). Pedagogia da esperança: um reencontro com a pedagogia do oprimido. Editora Paz e Terra.

Kiya, M. C. D. S. (2014). O uso de Jogos e de atividades lúdicas como recurso pedagógico facilitador da aprendizagem. Os desafios da escola pública paranaense na perspectiva de professor PDE: Cadernos Didáticos Pedagógicos. Ortigueira, 2, 6-45.

Marin, J. C., \& da Costa Güllich, R. I. (2015) Estratégias do Pibid: jogos didáticos no ensino de Ciências e Biologia. Anais do SEPE - Seminário de Ensino, Pesquisa e Extensão. Vol V.

Mendes, K. D. S., Silveira, R. C. C. P., \& Galvão, C. M. (2008). Revisão integrativa: método de pesquisa para a incorporação de evidências na saúde e na enfermagem.

Miranda, J. C., Gonzaga, G. R., \& Costa, R. C. (2016). Produção e avaliação do jogo didático" tapa zoo" como ferramenta para o estudo de zoologia por alunos do ensino fundamental regular/production and evaluation of a educational game" tapa zoo" As a tool for zoology study for students of regular elementary school. olos, 32(4), 383.

Pinto, L. Q., Pais, A. C. V. B., Nóbile, F. H. M., Gabriel, G. M., \& Sodero, J. P. T. (2021). Descobrindo os Elementos: a elaboração de jogos didáticos como alternativa de ensino. Brazilian Journal of Development, 7(1), 2247-2253.

Salido López, P. V. (2020). Metodologías activas en la formación inicial de docentes: Aprendizaje Basado en Proyectos (ABP) y educación artística.

Salomão, H. A. S., Martini, M., \& Jordão, A. P. M. (2007). A importância do lúdico na educação infantil: enfocando a brincade ira e as situações de ensino não direcionado. Portal de psicologia. 
Research, Society and Development, v. 10, n. 4, e41210414309, 2021

(CC BY 4.0) | ISSN 2525-3409 | DOI: http://dx.doi.org/10.33448/rsd-v10i4.14309

Silva, J. N. D. C. (2013). A importância da ludicidade no ensino de ciências. Monografia. Universidade Federal da Paraíba - UFPB Virtual. Curso de Licenciatura em Ciências Biológicas..

Sossela, G. C. S., \& Crocetti, S. (2013). Jogos como facilitadores do ensino de biologia. Revista Virtual Desafios da Escola Pública Paranaense na Perspectiva do Professor PDE-Paraná, 1 . 